\title{
Politische Kultur aus der Sicht der empirischen Sozialforschung
}

\author{
Oscar W. Gabriel
}

\section{Politischen Kultur im vereinigten Deutschland - eine Herausforderung für die empirische Politikforschung}

Auf dem Hintergrund des in Deutschland vorherrschenden Verständnisses von politischer Kultur muß es als ein erstaunlicher Tatbestand angesehen werden, daß der Beitritt der fünf neuen Länder zur Bundesrepublik keinen Boom an Publikationen über die kulturelle Herausforderung durch das Experiment Wiedervereinigung auslöste. Allerdings eignet sich der Beitritt der fünf neuen Länder nicht nur als Stimulans für eine volkspädagogisch motivierte Verwendung des Konzeptes „politische Kultur“, er konfrontiert auch die empirische Politikforschung mit interessanten Fragen; denn nach dem Beitritt fünf neuen Länder befindet sich die Bundesrepublik in einem strukturellen und kulturellen Umbruch, dessen Ergebnis noch im ungewissen bleibt.

Die Schaffung eines gesamtdeutschen Staates und einer gemeinsamen Verfassung für diesen Staat stellt nur einen ersten Schritt im Prozeß der Integration Deutschlands dar. Der Erfolg oder das Scheitern dieses Unternehmens wird nicht zuletzt von der Entwicklung der politischen Kultur abhängen. $\mathrm{DaB}$ mit demokratischen Strukturen nicht automatisch eine demokratische politische Kultur entsteht, hatte Sidney Verba (1965, S. 517) bereits vor fast 30 Jahren festgestellt: „A new constitution, ..., will be perceived and evaluated in terms of the political culture of a people. When put into practice in one society it may look quite different from the same constitution instituted in another nation with another political culture. ... History is full of examples of constitutions that did not 'take' as the constitution writer had hoped because their application was mediated through a particular political culture...". Auf dem Hintergrund der vorliegenden Erkenntnisse über die Entsprechung von politischer Struktur und politischer Kultur kann man Jesses (1992, S. 133ff.) Annahme, nach der Wiedervereinigung sei die Bundesrepublik größer, aber nicht anders geworden, allenfalls den Status einer empirisch zu prüfenden Hypothese zubilligen. Die Frage, ob sich in der Bundesrepublik Deutschland die kulturellen Grundlagen eines funktionsfähigen demokratischen Regimes herausbilden werden, ob mit der Spaltung der politischen Kultur in zwei gegensätzliche, zumindest aber unterschiedliche Subkulturen zu rechnen ist und ob die für die alten Ländern bisher typischen politischen Orientienungen fortbe- 
stehen werden, wird in den kommenden Jahren die empirische Politikforschung beschäftigen.

Empirische Untersuchungen der politischen Kultur können an eine gut etablierte internationale Forschungstradition anknüpfen. Die Aufgabe dieses Beitrages besteht darin, die Fragestellungen und Probleme bei der empirischen Analyse der politischen Kultur Deutschlands zu demonstrieren. Im einzelnen sind die folgenden Fragen zu klären:

(1) Welcher Ausschnitt aus der politischen Wirklichkeit konstituiert den Gegenstand der empirischen Analyse politischer Kultur und wie läßt sich dieser Realitätssauschnitt für die empirische Forschung erschließen?

(2) Welche konkreten Einzelfragen stehen bei der Analyse der kulturellen Entwicklung Gesamtdeutschlands im Vordergrund?

(3) Welche Strategien sind in der empirischen Forschung einzusetzen?

\section{Das Konzept der politischen Kultur in der empirischen Politikforschung}

Seit den fünfziger Jahren entwickelte sich die Analyse politischer Kultur zu einem der populärsten politikwissenschaftlichen Forschungsgegenstände. Wie Almond (1987, S. 28) mit Blick auf die amerikanische Politikwissenschaft ausführt, ,ist es offensichtlich, daß das politische Kultur-Konzept Eingang in das gängige Vokabular der Politikwissenschaft gefunden hat. Es ist Bestandteil allgemeiner Erklärungsansätze im Fach geworden. Es gibt $A n l a ß$ zu einer andauernden Diskussion in der Disziplin, die zwar nicht so umfangreich wie die Pluralismus-Debatte, aber im quantitativen Sinne doch sehr beachtlich ist. Es gibt etwa 35 bis 40 Abhandlungen empirischer oder theoretischer Art zur politischen Kultur im Buchformat und mehr als 50 Beiträge zu Fachzeitschriften und Symposien. Die Zahl der Verweise und Fußnoten in der Literatur übersteigt Eintausend" (vgl. auch Patrick, 1984; für die Bundesrepublik Deutschland Iwand, 1985).

Für die außerordentliche Popularität des Konzeptes „politische Kultur“ war allerdings ein hoher Preis zu entrichten. Wegen der weitgehend beliebigen Verwendung des Begriffs charakterisierte Kaase (1983) die wissenschaftiche Auseinandersetzung mit politischer Kultur als Versuch, einen Pudding an die Wand zu nageln. Nach Dias (1971, S. 409f.) deckt das Konzept so unterschiedliche Sachverhalte ab wie „Ideologie, Werte, politische Prädispositionen, Wertorientierung, normative innere Ordnung, modale nationale Persönlichkeit, politische Identität, politisches Erwartungssystem, fundamentale Übereinstimmung über Art und Stil politischer Handlung, fundamentale politische Orientierungen, politische Handlungen, Meinungen, Normen, Symbole, politisches Verhalten, politische Persönlichkeit usw.“. Eine seriöse empirische Analyse der Eigenschaften und der Entwicklung der politischen Kultur eines Landes setzt demnach eine präzise Festlegung des Gegenstandsbereichs der Analyse politischer Kultur und der dabei anzuwendenden Methoden voraus, die zumindest den folgenden Anforderungen genügt: 
(1) Das Konzept der politischen Kultur muß einen von anderen politikwissenschaftlichen Konzepten eindeutig abgrenzbaren Realitätsausschnitt bezeichnen.

(2) Das Konzept und die aus ihm ableitbaren theoretischen Konstrukte müssen einer empirischen Operationalisierung und Prüfung zugänglich sein.

(3) Die in der empirische Analyse benutzen Indikatoren müssen theoretisch begründet, valide und reliabel sein.

(4) Der mit dem Begriff politische Kultur bezeichnete Komplex von Variablen muß einen eigenständigen Beitrag zur Erklärung politischer Wirklichkeit leisten.

\subsection{Gegenstandsbestimmung}

Die ersten Analysen politischer Kultur entstanden im Kontext der behavioralistischen Neuorientierung der Politikwissenschaft. Deren Ziel bestand u.a. darin, das empirisch beobachtbare Verhalten politischer Akteure sowie dessen Korrelate ins Zentrum der Forschung zu rücken (vgl. ausführlich Falter, 1980). Bei der Auseinandersetzung mit den Bestimmungsfaktoren des politischen Verhaltens kamen auch die subjektiven oder psychologischen Aspekte der Politik, die Vorstellungen der Menschen von der Politik, in den Blick (vgl. Verba, 1965, S. 516).

In „The Civic Culture" unterbreiteten Almond und Verba (1965, S. 13) den Vorschlag, als politische Kultur "the particular distribution of patterns of orientation towards political objects among the members of the nation" zu bezeichnen. Weitere Informationen, insbesondere über die Bedeutung der politischen Kultur für das $\mathrm{Zu}$ sammenleben von Menschen, enthält eine Arbeit von Pye (1972, S. 293), in der die politische Kultur als Menge von Haltungen, Glaubensüberzeugungen und Gefühlen charakterisiert wird, die dem politischen Prozeß Ordnung und Sinn gäben. Sie bezögen sich auf die grundlegenden, verhaltensbestimmenden Gegebenheiten und Regeln, die Ideale und Verfahrensnormen der Politik.

Aus diesen Aussagen ergeben sich einige wichtige Eigenschaften des für die empirische Forschung typischen Verständnisses politischer Kultur:

(1) Die politische Kultur eines Landes ist über die Orientienungen oder Einstellungen von Individuen zu erfassen.

(2) Diese Einstellungen beziehen sich im weiteren Sinne auf alle politischen Objekte (Almond \& Verba, 1965), im engeren Sinne auf die grundlegenden, verhaltensbestimmenden politischen Sachverhalte (Pye, 1972). Durch den Bezug auf die Politik unterscheidet sich die politische Kultur von anderen Aspekten der Kultur einer Gesellschaft, z.B. von den Einstellungen zur Wirtschaftsordnung, zu Wissenschaft und Technik usw. Art und AusmaB der Wechselbeziehungen zwischen der politischen Kultur und anderen Aspekten des Kultursystems einer Gesellschaft können empirisch untersucht werden.

(3) Wie das Definitionsmerkmal „Verteilung von Einstellungen“ erkennen läßt, weisen die zu einem politischen Kollektiv gehörenden Individuen nicht notwendigerweise einheitliche politische Einstellungen auf. Die Feststellung des AusmaBes an Homogenität oder Heterogenität einer politischen Kultur ist ein Problem der empirischen Analyse. 
(4) Einstellungen erfüllen in der Auseinandersetzung des Individuums mit seiner Umwelt spezifische Funktionen. Sie dienen der Wahrnehmung (,cognitive map“) oder der Bewertung der politischen Umwelt (,normative map“; vgl. Beer, 1973, S. 23ff.) sowie der eigenen Rolle in dieser, disponieren zu bestimmten Formen des Verhaltens und beeinflussen - vermittelt über das politische Verhalten - das Funktionieren des politischen Systems.

(5) Die Orientierungen bilden ein Muster, d.h. sie weisen ein Mindestmaß an Interdependenz und an Beständigkeit auf.

(6) Die Verteilung der individuellen politischen Orientierungen in einem Kollektiv macht dessen politische Kultur aus. Die Einstellungen als Individualmerkmale betreffen die Mikro-Dimension der Politik, der Begriff Kultur bezieht sich auf die Meso- oder Makro-Dimension der Politik. Nicht Individuen, sondern Kollektive wie Nationen, Regionen, Gemeinden, Klassen oder Religionsgemeinschaften haben eine Kultur. Jedoch haben Individuen durch ihre Einstellungen an der Kultur eines Kollektivs teil.

Zu diesem von Patrick (1984) als „einstellungsbezogen“ bezeichneten Konzept politischer Kultur wurden mehrere Alternativen entwickelt, die die Autorin als ,objektiven“, „umfassenden“ und „heuristischen“ Ansatz charakterisierte. Diese Differenzierung bezieht sich auf den Gegenstand und den Theoriestatus der Konzepte. Während der psychologische Ansatz im Sinne Parsons' (et al. 1962, passim) Kultur als System von Orientierungen begreift, betont der objektive Ansatz die zweite Dimension des Parsonsschen Kulturkonzeptes und versteht Kultur als System kultureller Objekte oder Symbole. Der umfassende Ansatz schließt beide Aspekte und zusätzlich das politische Verhalten sowie weitere Ausschnitte aus der politischen Wirklichkeit ein. Im Hinblick auf den Theoriestatus kann man zwischen solchen Konzepten politischer Kultur unterscheiden, die explizit in eine Theorie eingebettet sind, und solchen, deren Interesse lediglich der Beschreibung konkreter Ausprägungen politischer Kulturen gilt.

Der von Patrick vorgeschlagene Ordnungsversuch läßt etliche Fragen offen. Es ist z.B. nur schwer nachvollziehbar, aus welchen Gründen Pye, von dem die Charakterisierung der politischen Kultur als psychologische Dimension der Politik stammt, als Vertreter des umfassenden Ansatzes figuriert. Nicht weniger irritierend ist die Zuordnung Eastons zum objektiven Ansatz; denn zahlreiche im Bezugsrahmen des psychologischen Ansatzes erstellte Untersuchungen greifen auf Eastons theoretische Konzepte zurïck. Schließlich überzeugt dieAbgrenzung zwischen dem heuristischenAnsatz einerseits und den drei verbleibenden Ansätzen nicht. Zwar könnte jeder der zuvor genannten Ansätze prinzipiell in eine politikwissenschaftliche Theorie integriert sein, mit Blick auf die tatsächliche Forschungssituation erscheint es jedoch angemessen, alle bisher entwickelten Konzepte als ,heuristisch“ einzustufen.

Als Grundlage der Klassifikation der Forschungsansätze reicht die Unterscheidung nach ihrem Objektbereich (einstellunsgorientiert, objektiv, umfassend) aus. In der empirischen Forschungspraxis spielt der einstellungsorientierte Ansatz die führende Rolle. Nachfolgend soll gezeigt werden, worin seine spezifischen Vorzüge für die empirische Forschung liegen und welche Probleme bei seiner Umsetzung in die Forschungspraxis auftreten. 


\subsection{Die Anwendung des einstellungsorientierten Konzeptes in der empirischen Forschung - Möglichkeiten und Probleme}

Die Dominanz des einstellungsorientierten Ansatzes in der empirischen Forschung entwickelte sich nicht zufällig, sondern aus theoretischen, wissenschaftsgeschichtlichen und forschungspraktischen Gründen.

Ausgehend von den Vereinigten Staaten nahm die empirische Einstellungsforschung seit dem Ende des Zweiten Weltkrieges einen bemerkenswerten Aufschwung. In diesem Kontext entstanden zahlreiche für die empirische Analyse politischer Kultur relevante Konzepte und zu ihrer Messung geeignete Instrumente (vgl. die Zusammenfassung bei Miller \& Traugott, 1989). In Bundesrepublik erreichte die empirische Umfrageforschung mittlerweile ebenfalls einen beachtlichen Grad an Professionalisierung (vgl. ZUMA, 1983). Auf Grund dieser Entwicklung stehen der einstellungsorientierten Analyse politischer Kultur bewährte, einer ständigen kritischen Diskussion ausgesetzte Forschungskonzepte und Meßinstrumente zur Verfügung. Ein vergleichbarer Grad an Professionalisierung ist bei konkurrierenden Ansätzen nicht zu erkennen.

Neben wissenschaftsgeschichtlichen und forschungspraktischen Erwägungen sprechen theoretische Argumente für die Konzeptualisierung politischer Kultur als Verteilung politischer Einstellungen in Kollektiven. Zunächst ist diese Vorstellung von politischer Kultur analytisch klar von anderen politikwissenschaftlichen Konstrukten, etwa von dem des politischen Verhaltens oder dem der politischen Institutionen, unterschieden. Hieraus ergibt sich aber nicht allein der Vorzug analytischer Klarheit. Darüber hinaus bietet sich auf diese Weise die Möglichkeit, den als politische Kultur bezeichneten Variablenkomplex in allgemeine politikwissenschaftliche Theorien zu integrieren. Die verhaltenstheoretische Politikforschung behandelt politische Einstellungen als Vermittlungsgrößen zwischen der sozialen Herkunft von Individuen und ihrem politischen Verhalten. Demnach wird das Verhalten einer Person nicht zuletzt von ihren Einstellungen bestimmt, die ihrerseits in einem Zusammenhang mit ihrem sozialen Kontext stehen (vgl. Falter, 1972). Darüber hinaus übt das Verhalten der Mitglieder eines politischen Kollektivs einen Einfluß auf die Funktionsfähigkeit und Persistenz der politischen Strukturen aus. Damit hängt der Zustand des politischen Systems unter anderem von der politischen Kultur des betreffenden Kollektivs ab. Auf der Makro-Ebene der politischen Analyse spielt die politische Kultur eine ähnliche Rolle wie die politischen Einstellungen in mikropolitischen Verhaltenstheorien (vgl. Lijphart, 1980).

Im Hinblick auf die Eindeutigkeit des Forschungsgegenstandes, die Möglichkeit zur Formulierung von Hypothesen über die Bedingungen und Folgen des Auftretens bestimmter kultureller Muster sowie die Operationalisienung der theoretischen Konstrukte eignet sich der einstellungsorientierte Ansatz für die vergleichende empirische Analyse. Gleichwohl sei auf einige mit der Verwendung dieses Ansatzes verbundene Probleme verwiesen, die auch bei einer Untersuchung der politischen Orientierungsmuster in Ost- und Westdeutschland auftreten:

(1) Eine verläßliche empirische Messung politischer Einstellungen erweist sich aus mehreren Gründen als schwierig. Als latente Eigenschaften sind Einstellungen einer 
direkten Messung nicht zugänglich. Da dies nur mittelbar möglich ist, werden bei der Erhebung Meßfehler wahrscheinlich. Dieses Risiko besteht besonders bei Einstellungen, die sich auf abstrakte, dem Individuum wenig vertraute Sachverhalte beziehen. Nach den Befunden der empirischen Forschung gehört die Politik zu den Lebensbereichen, die viele Personen als abstrakt, kompliziert und undurchschaubar, vom eigenen Leben relativ weit entfernt und deshalb unwichtig einstufen. Politische Einstellungen dürften für diese Personen den Status relativ beliebiger und kurzfristig austauschbarer "non-attitudes“ aufweisen. Da die Bevölkerung Ostdeutschlands erst seit kurzer Zeit über Erfahrungen im Umgang mit einer pluralistischen Demokratie verfügt, werden non-attitudes im östlichen Teil Deutschlands vermutlich noch häufiger auftreten als im Westen. Der Ost-West-Vergleich wird also unter methodischen Gesichtspunkten problematisch. Allerdings lassen sich diese Schwierigkeiten nicht durch einen Verzicht auf Einstellungsuntersuchungen umgehen, sondern nur durch eine problembewußte empirische Forschung. Zudem trägt die empirische Messung der für den objektiven Ansatz charakteristischen Verwendung politischer Symbole zumindest ebenso große Fehlerrisiken in sich wie die Einstellungsmessung.

(2) Der Begriff „politische Einstellung“ bezieht sich auf eine heterogene Klasse politischer Sachverhalte. Einerseits deckt er oberflächliche, situations- oder personenbezogene Gefühle, wie die Erregung über einen Skandal oder die Abneigung gegen einen bestimmten Politiker, ab. Auf der anderen Seite umfaßt er grundlegende Wertorientierungen und ideologische Dispositionen wie die Unterstützung demokratischer Spielregeln, die Toleranz gegenüberAndersdenkenden usw. Almonds und Verbas klassische Definition politischer Kultur als „Muster individueller Orientierungen auf politische Objekte" gibt dem Forscher keine Kriterien an die Hand, die ihn zur Unterscheidung zwischen zentralen und peripheren politischen Einstellungen befähigen. Solange sich die Einstellungen auf das System als allgemeines Objekt, dessen Inputund Output-Aspekte richten, sind sie für die Analyse politischer Kultur relevant.

$\mathrm{Da}$ diese Situation unter theoretischen und forschungspraktischen Gesichtspunkten als unbefriedigend einzuschätzen ist, unterbreitete Patrick (1984, S. 292ff.) den Vorschlag, die Analyse der politischen Kultur auf grundlegende, strukturbestimmende Orientierungen zu beschränken. Weniger fundamentale und stark situationsbestimmte Einstellungen möchte sie als „Öffentliche Meinung" (,public opinion") bezeichnet und konzeptuell von der politischen Kultur getrennt wissen. Zu den Bestandteilen der politischen Kultur zählt sie die Einstellungen zur Struktur und Funktion des Herrschaftsapparates sowie zur Reichweite und zu den Grenzen politischer Macht. Noch erheblich restriktiver fällt Pappis (1986, S. 282) Empfehlung aus, politische Kultur definitorisch und forschungspraktisch mit dem „Legitimitätseinverständnis mit der Herrschaftsordnung eines sozialen Systems" gleichzusetzen.

Die von Pappi und von Patrick angesprochenen Einstellungen gehören ohne Frage zur wissenschaftlichen Analyse politischer Kultur. Allerdings wirft die von beiden Forschern vorgeschlagene Begrenzung des Forschungsfeldes mehr Probleme auf als sie löst. Selbst wenn die Annahmen über die Zentralität einzelner politischer Einstellungen theoretisch begründbar wären, würde damit ihre empirische Prüfung keineswegs überflüssig. Zudem ändern sich politische Einstellungen im Laufe der Zeit. Deshalb ist nicht auszuschließen, daß eine zum Zeitpunkt $t$ zentrale Orientierung zum 
Zeitpunkt $t+1$ zu den peripheren Elementen des Orientierungssystems gehört. Schließlich ist Zentralität ein relationaler Begriff. Nur auf der Basis einer empirischen Analyse der zwischen mehreren Orientierungen bestehenden Beziehungen lassen sich Aussagen über die Struktur des individuellen Überzeugungssystems machen. Die Differenzierung zwischen zentralen und peripheren Orientierungen ist somit kein Definitionsproblem, sondern eine Aufgabe der empirischen Analyse. Dies setzt aber voraus, $\mathrm{daB}$ alle politischen Orientierungen Gegenstand der Untersuchung sind.

Schon unter dem Gesichtspunkt ihrer systemischen Konsequenzen in der neueren deutschen Geschichte ist die empirische Analyse des Entstehens von Legitimitätsüberzeugungen in den neuen Ländern zwingend (vgl. zur Begründung Lipset, 1969). Eine Begrenzung der empirischen Analyse politischer Kultur auf diese Aspekte läßt sich aber weder theoretisch noch wissenschaftsgeschichtlich rechtfertigen. In politischen Systemen mit einer gefestigten Legitimität wird die Beziehung der Bevölkerung zur Politik von anderen Faktoren bestimmt, z.B. vom Vertrauen zur amtierenden Regierung, von der Bewertung des Outputs der politischen Institutionen und den Leistungen politischer Akteure. Auch unter diesem Gesichtspunkt ist eine Begrenzung der Analyse politischer Kultur auf Legitimitätsüberzeugungen nicht vertretbar.

Eine andere Forschungsstrategie als Patrick (1984) und Pappi (1986) schlägt Rohe (1987, S. 40) vor. Er bezeichnet die politische Kultur als „Muster von Vorstellungen, nicht von Einstellungen .... Wer politische Kulturforschung betreibt, will nicht wissen, ob und inwieweit ein politisches Regime und politische Institutionen bejaht oder abgelehnt werden, er interessiert sich vielmehr für die Prinzipien, die dieser Bejahung oderAblehnung zugrundeliegen". An anderer Stelle definieren Rohe und Dömer (1990, S. 24) politische Kultur als ,historisch gewachsenes, kollektiv geteiltes, den Individuen zumeist selbstverständliches und deshalb zumeist unbewußtes 'design for political living'.., das das Wahrnehmen, Denken, Fühlen und indirekt auch Handeln der politischen Akteure steuert".

Allerdings thematisiert Rohe damit weder völlig neue Forschungsprobleme noch eine Alternative zum einstellungsorientierten Ansatz. Die Vorstellung von politischer Kultur als System (Muster) historisch gewachsener, verhaltensrelevanter, zum Teil unbewußter kollektiver Orientierungen läßt sich ohne Probleme in die von Almond und Verba begründete Forschungstradition einbinden. Auch die in der Definition geforderte Ersetzung des Einstellungsbegriffs durch den der Vorstellungen erscheint nur auf den ersten Blick als radikaler Schnitt. In völliger Übereinstimmung mit Almonds und Verbas Charakterisierung politischer Kultur als Muster von Orientierungen ergibt sich aus der Definition die Forderung, die Struktur des gesamten Raumes politischer Einstellungen zum Gegenstand der Forschung zu machen. Dies ist in erster Linie als eine gerechtfertigte Kritik an der empirischen Forschungspraxis zu verstehen, die allzu sehr auf die Beschreibung einzelner Elemente des Orientierungssystems fixiert ist und die Muster der zwischen den einzelnen Orientierungen bestehenden Beziehungen vernachlässigt. Jedoch macht die Beschäftigung mit Einstellungsstrukturen die Erhebung einzelner Einstellungen nicht überflüssig, sondern setzt sie geradezu voraus. Die Fragen, ob die politischen Einstellungen von Individuen eine Struktur aufweisen und bei welchen Personengruppen dies der Fall ist, lassen sich wieder nur empirisch klären. 
(3) Ein drittes Problem bei der Anwendung des einstellungsorientierten Konzeptes ist forschungspraktischer Natur: Die tatsächliche Datenlage setzt historischen und international vergleichenden Analysen der politischen Kultur enge Grenzen. Größere nationale - und erst recht international vergleichende - Datenbestände liegen nur für die Zeit nach dem Zweiten Weltkrieg vor. In besondererem Maße gelten derartige Datenrestriktionen für den Vergleich der politischen Orientierungen in Ost- und Westdeutschland. Aus naheliegenden Gründen sind für die ostdeutsche Vorwendezeit kaum brauchbare Daten vorhanden. Soweit in der ehemaligen DDR Einstellungsforschung betrieben wurde, orientierte sie sich nicht an der internationalen Diskussion. Längerfristig angelegte vergleichende Untersuchungen des politisch-kulturellen Wandels in Deutschland, vergleichende Langzeitstudien von Sozialisationsprozessen usw. scheitern an der Datenlage. Als Startpunkt für vergleichende Untersuchungen der politischen Einstellungen in Ost- und Westdeutschland kommt das Jahr 1990 in Betracht, in dem erstmals im theoretischen Bezugsrahmen des political-culture Ansatzes stehende Umfragen durchgeführt wurden. Für die davor liegende Zeit dürfte auch der Rückgriff auf inhaltsanalytisch zu erfassende Ersatzdaten kaum in Frage kommen (vgl. dazu auch Rohe \& Dörner, 1990); denn in Anbetracht des raschen Zusammenbruchs der ehemaligen DDR sind Zweifel an der Annahme angebracht, offizielle Dokumente könnten irgendwelche verläßlichen Informationen über die tatsächlichen politischen Orientierungen der Bevölkerung liefern.

(4) Wegen des Datenproblems ist eines der zentralen Anliegen der Analyse politischer Kultur, die empirische Untersuchung des Einflusses der politischen Kultur auf die Stabilität und Performanz politischer Systeme, nicht realisierbar. Abgesehen von den mit der Operationalisierung der Begriffe „politische Stabilität“ oder „Performanz der politischen Institutionen“ verbundenen Schwierigkeiten (vgl. hierzu die wenig befriedigenden Ansätze bei Lipset, 1969; Powell, 1983), treten die für eine Untersuchung der systemischen Effekte kultureller Orientierungen erforderlichen Explanandum-Ereignisse, grundlegende Systemtransformationen, nur sehr selten auf. Sind sie beobachtbar, dann scheitert die Prüfung der Political Culture-Hypothese nicht allein an der geringen Zahl theorierelevanter Ereignisse, sondern darüber hinaus am Fehlen der für eine Erklärung benötigten Einstellungsdaten aus der Zeit vor der Systemtransformation. Auch wenn die von der Analyse politischer Kultur angestrebte, auch in unserem Falle hochinteressante Verknüpfung von Mikro- und Makroanalyse praktisch nicht durchführbar ist, werden die - praktikablen - mikropolitischen Analysen keineswegs überflüssig.

Trotz dieser Restriktionen erweist sich die einstellungsorientierte Analyse politischer Kultur in konzeptueller und methodischer Hinsicht nach wie vor als ein sinnvoller Weg. Sie steht in einer gut etablierten Forschungstradition, bezieht sich auf einen eindeutig eingegrenzten Objektbereich, verfügt über bewährte Erhebungsmethoden und läßt sich mit politikwissenschaftlichen, soziologischen und psychologischen Theorien verknüpfen. Auch aus einem direkten Vergleich ihres bisherigen Ertrages mit dem konkurrierender Ansätze ergibt sich kein Anlaß, die einstellungsorientierte Analyse politischer Kultur zu Gunsten eines anderen Vorgehens aufzugeben. 


\section{Forschungsgegenstände}

In „The Civic Culture“ hatten Almond und Verba (1965, S. 16ff.) ein Raster zur Beschreibung politischer Kulturen entwickelt und als Grundlage ihrer empirische Untersuchung benutzt. Es läßt sich aus der Definition politischer Kultur ableiten und enthält die Merkmalsdimensionen Orientierungsarten, Orientierungsobjekte und Muster der Verteilung von Orientierungen. Da die Differenzierung nach Orientierungsarten konzeptuell umstritten, schwer in die empirische Analyse umsetzbar und zudem für die Beschreibung moderner politischer Kulturen bedeutungslos ist (vgl. Einzelheiten bei Gabriel 1986, S. 62ff.), beziehen sich die folgenden Ausführungen ausschließlich auf die Orientierungsobjekte und die Verteilung der Orientierungen.

In Anlehnung an Eastons Systemtheorie gruppierten Almond und Verba (1965, S. 16ff.) die politischen Objekte zu vier Komplexen, die sie als System im allgemeinen, Inputs, Outputs und Egos Rolle als aktiver Teilnehmer im politischen Prozeß bezeichneten. Die Orientierungen der Bevölkerung auf diese Objekte können überwiegend positiv, negativ oder indifferent ausfallen. Ebenso kann das Ausmaß an Übereinstimmung objekt-, situations- und populationsspezifisch variieren. In der einschlägigen Literatur wurde bisher keineswegs zufriedenstellend geklärt, wie das Ausmaß an $\mathrm{Zu}$ stimmung zu den einzelnen Aspekten des politischen Systems dessen Stabilität und Funktionsfähigkeit beeinflußt und wie hoch der Konsensbedarf bzw. das Ausmaß an systemverträglichem Dissens ausfallen kann (vgl. die unterschiedlichen Überlegungen hierzu bei Almond \& Verba 1965, S. 192ff. und 341ff.; Döring, 1990; Gabriel, 1993; Parry, 1976; Sniderman, 1981; Wright, 1976).

Als Grundlage für die empirische Analyse bleibt die Unterscheidung zwischen System-, Input- und Outputorientierungen zu allgemein. Eine theoretisch begründete Präzisierung dieser abstrakten Konstrukte ist beim gegenwärtigen Forschungsstand nicht möglich. Dennoch kann man einige Kriterien für die Auswahl der Einstellungen angeben, die in eine empirische Analyse der politischen Kultur Gesamtdeutschlands einbezogen werden sollten.

(1) Die Untersuchung sollte diejenigen Einstellungen einschließen, deren konzeptueller Status in der Theoriediskussion und in der empirischen Forschung relativ gut geklärt ist und deren Analyse sich somit möglichst gut in einen etablierten Forschungskontext einfügt.

(2) Die Literatur enthält plausible Vermutungen über die spezifischen Merkmale der politischen Kultur Deutschlands, die Bedingungen ihres Zustandekommens und ihre Auswirkungen auf die Funktionsfähigkeit des politischen Systems, die der empirischen Forschung über den kulturellen Wandel in Gesamtdeutschland nützliche Orientierungshilfen bieten. Besondere Aufmerksamkeit verdient das Studium derjenigen Einstellungen, die als Ursachen für die Schwierigkeiten bei der Etablierung demokratischer politischer Strukturen in Deutschland angesehen wurden. Die neuen Länder stehen derzeit ebenfalls vor dem Problem, den im institutionellen Bereich bereits vollzogenen Systemwandel kulturell abzusichern. Insofern können die Erkenntnisse über den kulturellen Wandel in der alten Bundesrepublik, auch wenn sie nicht mechanisch auf die aktuelle Situation in Ostdeutschland übertragen werden können, die Auswahl von Untersuchungsproblemen und die Hypothesenbildung anleiten. 


\subsection{Die Systemdimension: Die Einstellungen zum politischen Regime und zur politischen Gemeinschaft}

Als das zentrale Merkmal der politischen Kultur eines Landes stufen Almond \& Verba (1965, S. 16) die Einstellungen zum System als allgemeines Objekt ein. Sie umfassen in Anlehnung an Easton (1979, vgl. auch Pappi, 1986) das Verhältnis der Bevölkerung zum politischen Regime und zur politischen Gemeinschaft. Als politisches Regime bezeichnet Easton (1979, S. 190ff.) die für das politische Zusammenleben von Menschen maßgeblichen Werte, Normen und Autoritätsstrukturen. Hierzu gehören unter anderem die Einstellungen zur Demokratie, zum Pluralismus, zu den Grundrechten und zur Gewaltenteilung. Als zentrale Elemente der politischen Kultur eines Landes beeinflussen die Regimeorientierungen die Beschaffenheit speziellerer politischer Einstellungen.Als zweitenAspekt der Systemkultur bezeichnet Easton (1979, S. 171ff.) die Einstellungen zur politischen Gemeinschaft, z.B. die Identifikation mit der Nation und ihren Symbolen sowie den anderen Mitgliedern des politischen Kollektivs.

Die Einstellungen der Bevölkerung zum politischen Regime und zur politischen Gemeinschaft fanden in der bisherigen Theoriediskussion und in der empirischen Forschungspraxis große Beachtung. Von grundlegender Bedeutung für die Untersuchung der Systemorientierungen waren die von Easton entwickelten Konzepte der diffusen und der spezifischen politischen Unterstïtzung sowie die damit verbundenen Konstrukte des politischen Vertrauens und der Legitimitätsüberzeugungen (vgl. u.a. Abramson, 1983; Fuchs, 1989; Westle, 1989). Besonders für die Untersuchung des Verhältnisses der Bevölkerung zum politischen Regime wurden in der internationalen Umfrageforschung Erhebungsinstrumente entwickelt, deren Anwendung in einer vergleichenden Analyse der politischen Kultur in Ost- und Westdeutschland möglich ist. Deren Einsatz macht es darüber hinaus möglich, die Regimeorientierungen der Bundesbürger in einen längerfristigen Entwicklungstrend einzuordnen und in international vergleichender Perspektive zu diskutieren. Theoretisch weniger gut fundiert und empirisch weniger breit angewandt wurden die Konzepte zur Messung der Einstellungen der Bevölkerung zur politischen Gemeinschaft (vgl. dazu Westle in diesem Band).

Ein Rückblick auf die politische Entwicklung Deutschlands seit dem Beginn der Neuzeit unterstreicht die wissenschaftliche und politisch-praktische Relevanz einer empirischen Analyse der Einstellungen der Bevölkerung zum politischen Regime und zur politischen Gemeinschaft: In der Vergangenheit hatte sich dieser Aspekt des Verhältnisses zwischen Bevölkerung und Politik nämlich immer wieder als problematisch erwiesen. Nach dem Bruch der Einheit von liberal-konstitutioneller und nationaler Bewegung befanden sich die Befürworter des nationalen Gedankens und die Anhänger demokratischer Ideale häufig in gegnerischen Lagem. Infolge der Teilung Deutschlands und der Diskontinuität demokratischer Regime blieb das Verhältnis der Deutschen zu Staat und Nation auch nach dem Zweiten Weltkrieg prekär. Eine positive Einstellung der Bevölkerung der alten Bundesrepublik zu den Werten, Institutionen und Verfahren einer liberal-pluralistischen Demokratie westlicher Prägung entwickelte sich nicht unmittelbar mit der Verabschiedung einer demokratischen Verfassung, sondern nur allmählich und in Konkurrenz mit alten Loyalitäten. Erst in dem Maße, indem die politischen Institutionen und Symbole der Vergangenheit ihre Anziehungs- 
kraft verloren, konnte in der Bundesrepublik die für eine funktionsfähige Demokratie charakteristische Systemunterstützung entstehen (vgl. Baker, Dalton \& Hildebrandt 1981, S. 22ff.; Conradt, 1980, S. 256ff.; dieselbe 1991, S. 134ff.; Fuchs, 1989; Gabriel 1987b).Zu den als problematischempfundenen und in ihrer Bedeutung zurückgedrängten Identifikationsobjekten dürfte auch das politische Symbol „Nation“" gehört haben.

Erschwert wurde die Entwicklung eines ungebrochenen Verhältnisses der Bundesbürger zur Nation sicher auch durch die nationale Teilung. Erst mit der Wiedervereinigung endete die vierzigjährige Phase einer feindlichen Koexistenz zweier souveräner Staaten, die zwar in einer gemeinsamen Tradition standen, nach 1945 jedoch immer enger in unterschiedliche Bündnissysteme und Wertegemeinschaften integriert wurden. Es ist zu vermuten, daß die langjährige Erfahrung der nationalen Teilung und die mit ihr verbundene Verpflichtung der beiden Teilstaaten auf gegensätzliche soziopolitische Ordnungsmodelle die politischen Einstellungen der Bevölkerung weiterhin beeinflussen werden.

Erste vergleichende Untersuchungen der Einstellungen der Bevölkerung beiderTeile Deutschlands zum politischen Regime und zur politischen Gemeinschaft wurden bereits unmittelbar nach der politischen Wende in der ehemaligen DDR durchgeführt. Sie brachten zum Teil überraschende Ergebnisse, zum Teil decken sie sich aber auch mit den Erwartungen. Auf dem Hintergrund der Entwicklung in den alten Bundesländern fällt die Übereinstimmung in der Unterstützung demokratischer Werte und Normen überraschend groß aus (vgl. Bauer, 1991; Feist, 1991; Gluchowski \& Zelle, 1992). Zugleich besteht in der Zufriedenheit mit den bestehenden politischen Strukturen und im Institutionenvertrauen ein beträchtliches West-Ost-Gefälle (vgl. Gabriel, 1993; Gluchowski \& Zelle, 1992). Diese Diskrepanz zwischen der Bewertung der Idee und der Realität der Demokratie unterscheidet die derzeitigen Regimeorientierungen in Ostdeutschland von der Einstellungsstruktur der Bundesbürger in den fünfziger Jahren. Trafen hier die Vorbehalte sowohl die demokratischen Ideale als auch deren Realisierung in der politischen Praxis, so steht in den neuen Ländern eine positive Einstellung zu den demokratischen Idealen einer weit verbreiteten Unzufriedenheit mit dem aktuellen Funktionieren des politischen Systems gegenüber. Darüber hinaus ist - im Unterschied zur politischen Situation der alten Bundesländer in den beiden ersten Nachkriegsjahrzehnten - im Osten der Bundesrepublik derzeit nur eine schwache Identifikation mit den politischen Strukturen und Symbolen der Vergangenheit festzustellen.

Die empirische Forschung wird in den kommenden Jahren zu klären haben, ob in der Bevölkerung der neuen Bundesländer Unterstïtzung für ein politisches System entsteht, dessen Institutionen, Werte und Normen einen Bruch mit der zuvor bestehenden politischen Ordnung darstellen oder ob die bestehenden politischen Rahmenbedingungen eine Fragmentierung der Systemorientierung entlang der ehemaligen Grenze zwischen der Bundesrepublik und der DDR begünstigen. Zudem ist zu prüfen, ob dieser Integrationsproze $B$ - wie in der alten Bundesrepublik - von relativ klar identifizierbaren gesellschaftlichen Gruppen getragen wird und welche Rolle die ökonomische Entwicklung in diesem Prozeß $B$ spielt. Angesichts der gravierenden wirtschaftlichen Probleme in Ostdeutschland und der erheblichen Diskrepanz zwischen den Lebensbedingungen im Osten und Westen der Bundesrepublik dürfte die Etablierung einer gemeinsamen „Systemkultur“ in Deutschland schwierig werden. 
Auch für den Westen Deutschlands, dessen politische Kultur gelegentlich als die einer „Schönwetterdemokratie“ charakterisiert wurde (vgl. Sontheimer, 1989, bes. S. 111ff.), ist im Hinblick auf die zu erwartenden Einschnitte in die sozialen Besitzstände ein Fortbestand der erreichten Systemakzeptanz keineswegs selbstverständlich. Zumindest auf mittlere Sicht könnte der Beitritt der neuen Länder im Westen einen Rückgang des Vertrauens zu den politischen Institutionen und zum gesamten politischen System nach sich ziehen. Wie Untersuchungen in anderen westlichen Demokratien zeigen, kann ein einmal erreichtes Vertrauensniveau durch perzipierte Leistungsdefizite oder Fehlentwicklungen im Bereich der symbolischen Politik verspielt werden (vgl. z.B. für die USA Abramson, 1983, S. 11ff. und S. 225ff.; Abramowitz, 1980; Lipset \& Schneider, 1983; für Großbritannien Kavanagh, 1980; Marsh, 1977; Topf, 1989). Auf dem Hintergrund der internationalen Diskussion über die Determinanten politischer Unterstützung wird vor allem der Einfluß der Regimeperformanz auf die Einstellungen der Bevölkerung zum politischen Regime und zur politischen Gemeinschaft empirisch zu untersuchen sein.

\subsection{Die Output-Dimension: Vertrauen zur Regierung und zur Exekutive}

Der Output-Komponente der politischen Kultur sind in Anlehnung an Easton (1979) die Einstellungen zu den regulativen Aktivitäten des politischen Systems zuzuordnen. Als Produzenten dieser Leistungen treten der Bevölkerung bestimmte Institutionen wie die Regierung, die Verwaltung, die Gerichte oder Parlamente gegenüber, deren Aktivitäten die Bürgerinnen und Bürger unter Nutzengesichtspunkten (,performance“) oder im Hinblick auf allgemeine, leistungsunabhängige Eigenschaften (,quality“, vgl. Parsons \& Shils, 1962, S. 76ff.) bewerten. Mit dem Regierungsvertrauen, der PolicyZufriedenheit (vgl. Muller \& Jukam, 1977) sowie der Untertanenkompetenz (vgl. Almond \& Verba, 1965) entwickelte die empirische Forschung einige in vergleichenden Untersuchungen eingesetzte Konzepte zur Analyse von Outputorientierungen.

Nach den Befunden der einschlägigen Forschung spielten die Outputorientierungen in Deutschland traditionell eine wichtige Rolle als Bindeglieder zwischen der Bevölkerung und dem Staat. Noch in der Civic Culture-Studie hattenAlmond \& Verba (1965, S. 47ff. und S. 169ff.) ein im internationalen Vergleich hoch entwickeltes Vertrauen zur Exekutive festgestellt. Auch Baker, Dalton und Hildebrandt (1981, S. 22ff.) und Conradt (1991) brachten den Aufbau von Systemunterstützung in der Bundesrepublik mit positiven Einstellungen zur Leistungsfähigkeit und Vertrauenswürdigkeit der amtierenden Regierung in Verbindung. Neuere Studien vermitteln in dieser Hinsicht ein ambivalentes Bild (vgl. z.B. Gabriel, 1987a, 1992, 1993).

Die positiven Einstellungen zur Bürokratie, zur Polizei, zu den Gerichten und zur Regierung scheinen in der Bundesrepublik der fünfziger Jahre die Integration der Bevölkerung in das neu geschaffene demokratische Regime gefördert zu haben. Hierfür waren mehrere Gründe maßgeblich: Einerseits begründeten die rasch spürbaren Erfolge beim wirtschaftlichen und gesellschaftlichen Wiederaufbau des Landes eine zunehmend positive Orientierung der Bevölkerung gegenüber der politischen Füh- 
rung und den politischen Institutionen insgesamt. Auf der anderen Seite bedeuteten die zwölf Jahre nationalsozialistischer Herrschaft für das Verhältnis der Bevölkerung zu den politischen Institutionen nur partiell einen Bruch mit der deutschen Tradition des autoritären Wohlfahrtsstaates. Große Teile des Rechts sowie der Exekutivorganisation wurden zwischen 1933 und 1945 beibehalten, und auch in personeller Hinsicht bestand - von der Spitze abgesehen - eine beträchtliche Kontinuität. Dies galt auch für die Organisation und Arbeitsweise der Exekutive in den fünfziger Jahren, die im Vergleich mit der Vorkriegszeit eher durch strukturelle und personelle Kontinuität als durch tiefgreifenden Wandel charakterisiert war. Die Strukturen der Willensbildung und Interessenvermittlung sowie der staatliche Entscheidungsapparat waren durch die Nationalsozialisten vollständig gleichgeschaltet worden. Dagegen hatten zumindest die unteren Verwaltungsinstanzen, über die ein erheblicher Teil der persönlichen Interaktionen zwischen der Bevölkerung und dem Staat abläuft, während der Zeit des Nationalsozialismus ein begrenztes $\mathrm{Ma} \beta$ an Autonomie bewahren können. In der Periode des Aufbaus eines neuen politischen Regimes bestand insofern die Möglichkeit, traditionelle Loyalitätsobjekte für den Aufbau von politischer Unterstützung für die neue politische Ordnung nutzbar zu machen.

Die aus der jüngsten Zeit vorliegenden Daten über die Einstellungen der Bundesbürger zu Regierung und Verwaltung stützen die Charakterisienung der politischen Kultur Deutschlands als Untertanenkultur nicht mehr, insbesondere im internationalen Vergleich.

Auf den ersten Blick mag es verlockend sein, in den Beziehungen der Bevölkerung zu den Outputinstitutionen Parallelen zwischen der alten Bundesrepublik der fünfziger Jahre und der aktuellen Situation in den neuen Ländern zu ziehen. Einige Studien vertreten die politische Kultur der ehemaligen DDR in der Tradition des preußischen Obrigkeitsstaates. Das politische System stellte sich für die Bevölkerung als allzuständige Schutz- und Ordnungsmacht dar, die das öffentliche und private Leben einer umfassenden staatlichen Kontrolle unterwarf, eine strikte Unterordnung unter die staatliche Autorität und die sie repräsentierenden Institutionen verlangte, Abweichungen von den offiziell genehmigten Verhaltensmustern streng ahndete, als Gegenleistung für die Einhaltung der Normen und Spielregeln der sozialistischen Demokratie jedoch eine umfassende Sicherung der individuellen Existenz garantierte (vgl. Grunenberg, 1989). Die Frage nach dem Erfolg der angestrebten Verbindung traditioneller preußischer Tugenden mit der sozialistischen Staats- und Gesellschaftsdoktrin läßt sich nicht sicher beantworten. Immerhin vermitteln nach der Wende durchgeführte Umfragen den Eindruck, daß die Werte und Normen eines autoritären Staates in den neuen Ländern eine wesentlich breitere Unterstützung fanden als in der alten Bundesrepublik (vgl z.B. Feist, 1991, S. 26).

Bei genauerem Hinsehen spricht allerdings wenig für die Annahme, daß die Outputorientierungen in den neuen Ländern eine ähnliche Rolle für die Unterstützung des politischen Systems spielen könnten wie es in den beiden ersten Nachkriegsdekaden in der alten Bundesrepublik der Fall war. Von der ökonomischen Entwicklung in den neuen Ländern gehen bislang keine systemlegitimierenden, sondern eher belastende Wirkungen aus. Die wirtschaftliche Restrukturierung einschließlich ihrer negativen Implikationen für den Arbeitsmarkt ist noch nicht abgeschlossen. Die wirtschaftliche 
Entwicklung wird im Osten dramatisch schlechter eingeschätzt als im Westen, und dies alles kollidiert mit der Forderung nach einer möglichst umgehenden Angleichung der Lebensverhältnisse im Osten an das Westniveau.

Anders als in der Bundesrepublik der fünfziger Jahre besteht auch keine Möglichkeit, eine positive Bindung an einzelne Institutionen des alten Regimes auf den veränderten Systemkontext zu übertragen. Das politische System der DDR verstand sich als Gegenmodell zur bürgerlich-kapitalistischen Demokratie des Westens und wurde dort auch so perzipiert. Über die Möglichkeit, Institutionen oder Rechtsnormen der ehemaligen DDR in die neue Bundesrepublik zu übernehmen, wurde nicht einmal diskutiert. In Anbetracht des nach der DDR-Gründung vollzogenen totalen Bruchs mit der deutschen Rechts- und Verwaltungstradition wäre dies allenfalls in peripheren Bereichen der Leistungsverwaltung möglich gewesen. Selbst wenn man in Rechnung stellt, daß die neuen Bundesländer nach der Wende einen großen Teil des Exekutivpersonals übernahmen, waren dieVerwaltungsstrukturen und Rechtsgrundlagen desVerwaltungshandels nach der Wende völlig andere als davor. Der mit der Wende in den neuen Ländern vollzogene Einschnitt in die Administrativorganisation war erheblich tiefer als in der Bundesrepublik der fünfziger Jahre. Eine mit der damaligen Situation vergleichbare Kontinuität in der Exekutivorganisation war nach dem Systemwechsel nicht vorhanden.

Auch wenn autoritär-obrigkeitsstaatliche Werte und Verhaltensdispositionen in den neuen Ländern noch relativ weit verbreitet sind, bedeutet dies nicht, daß sich mit ihnen eine positive Einstellung in die Leistungsfähigkeit und Vertrauenswürdigkeit von Exekutivinstitutionen verbindet. Vielmehr zeigen erste vergleichenden Untersuchungen des Institutionenvertrauens in den alten und neuen Bundesländern gerade bei den Exekutivinstitutionen ein gravierendes Vertrauensgefalle zwischen dem Westen und dem Osten der Bundesrepublik (vgl. Feist, 1991, S. 26ff.; Gabriel, 1993; Gluchowski \& Zelle, 1992; Rattinger, 1993, S. 26ff.). Trotz eines gewissen Vertrauensgewinns im Zeitraum 1991/92 fungieren derzeit in den neuen Bundesländern weder die Gerichte noch die Polizei als Kristallisationskerne für das Entstehen von Systemvertrauen, und erst recht gilt dies für die Regierung, die seit der Wende in den neuen Ländern einen drastischen Vertrauensverlust hinnehmen mußte. Es wird zu prüfen sein, ob sich in den neuen Ländern mit einer Normalisierung der sozio-ökonomischen Bedingungen eine Änderung im Verhältnis der Bevölkerung zur Exekutive einstellen wird. Aus den bereits bekannten Gründen verdient allerdings auch die weitere Entwicklung in den alten Bundesländem Interesse.

\subsection{Die Input-Dimension: Partizipative Orientierungen}

Die Input-Dimension betrifft die partizipative Komponente der Politik, d.h. die Art und Weise, in der Individuen, Gruppen und Organisationen politische Interessen artikulieren, politische Unterstützung gewähren und entziehen sowie politischen Einfluß ausüben. In diesen Prozessen werden bestimmte Aktivitäten ausgeführt, Rollen, Institutionen, Organisationen, Spielregeln und Verfahren entwickelt, die den Zugang zum politischen System möglich machen und regeln. Erst mit der Ausdifferenzierung lei- 
stungsfähiger Input-Strukturen vollzieht ein politisches System den Übergang von einem vordemokratischen zu einem demokratischen Stadium; denn zu einer modernen Demokratie gehören Institutionen und Prozesse, die es der Bevölkerung ermöglichen, eine aktive Rolle in der Politik zu spielen (vgl. Dahl, 1971).

Mit der Wahrnehmung und Bewertung partizipativer Strukturen durch die Bevölkerung bildet die politische Kultur eine partizipative Dimension aus. Die Individuen nehmen ihre eigene Rolle als Teilnehmer am politischen Leben sowie die damit verbundenen Rechte und Pflichten wahr und perzipieren ihre politische Umwelt im Hinblick auf deren Offenheit für die Belange der Bevölkerung. Die partizipative Komponente der politischen Kultur betrifft also das Selbstverständnis der Bürgerinnen und Bürger als potentielle Teilnehmer am politischen Prozeß sowie eine geistige Auseinandersetzung mit den institutionell-organisatorischen Vorkehrungen, die diese Teilnahme am politischen Leben möglich machen.

Almond \& Verba (1965, S. 136ff.) hatten die partizipative Komponente der politischen Kultur über das Konzept der subjektiven politischen Kompetenz erfaßt. Damit bezeichneten sie Überzeugungen von Individuen, im Bedarfsfalle (erfolgreich) auf politische Vorgänge Einfluß nehmen zu können. Als ein konzeptuell und methodisch der subjektiven politischen Kompetenz ähnliches Konzept führte die Forschergruppe um Angus Campbell (1954) das Gefühl politischer Effektivität in die empirische Forschung ein. In Anschlußuntersuchungen wurde eine Differenzierung zwischen interner und externer Effektivität vorgeschlagen. Wie das Gefühl subjektiver politischer Kompetenz deckt erstere das politische Selbstbewußtsein von Individuen ab, letztere betrifft die Überzeugung, daß die bestehenden politischen Institutionen eine Einflußnahme des Durchschnittsbürgers auf die Politik zulassen und daß die Inhaber politischer Führungspositionen einer derartigen Einflußnahme zugänglich sind (vgl.Abramson, 1983, S. 11ff. und S. 225ff.). Ebenfalls im Kontext der Ann-Arbor-Gruppe entstand das Konzept der Parteiidentifikation als Instrument zur Erfassung der Beziehung der Bevölkerung zu den politischen Parteien als den bedeutsamsten Akteurseinheiten im Input-Prozeß (vgl. den Beitrag Rattinger in diesem Band).

Die Konzepte der politischen Kompetenz, der politischen Effektivität und der Parteienidentifikation konnten sich in der internationalen empirischen Forschung fest etablieren. In zahlreichen Beiträgen wurde die theoretische Konzeptualisierung diskutiert, wurden Vorschläge zur empirischen Messung dieser Einstellungen unterbreitet und vergleichende empirische Analysen durchgefuihrt (vgl. die Hinweise bei Gabriel 1986, S. 195ff.). Zwar deckt die Analyse dieser Einstellungen keineswegs das gesamte Spektrum der Input-Orientierungen ab, jedoch bezieht sie sich auf zentrale Forschungsfelder, zu deren Erfassung gut fundierte theoretische Konzepte und bewährte Meßinstrumente vorliegen.

Für die Berücksichtung der genannten Variablen in einer vergleichenden empirischen Analyse der politischen Orientierungsmuster in Ost- und Westdeutschland sprechen bestimmte historische Tatbestände. Nach den vorliegenden Befunden der empirischen Forschung entstanden die für eine Civic Culture typischen partizipativen Orientierungen in Deutschland erst sehr spät. Noch Ende der fünfziger Jahre konstatierten Almond \& Verba in der Bundesrepublik ein im internationalen Vergleich schwach entwickeltes Gefühl subjektiver politischer Kompetenz (1965, S. 136ff.). Auch Baker, 
Dalton \& Hildebrandt (1981, S. 28) bezeichneten das Effektivitätsgefühl der Deutschen als „alarmingly low“. In den siebziger und achtziger Jahren durchgeführte Erhebungen deuten zwar auf ein gestiegenes staatsbürgerliches Selbstbewußtsein hin (vgl. Baker, Dalton \& Hildebrandt, 1981, S. 22ff.; Conradt 1980, S. 256ff.; Gabriel, $1986,1987,1990$ ), jedoch glaubt nach wie vor nur eine Minderheit unter den Bundesbürgern, in Fragen der nationalen Politik politischen Einfluß ausüben zu können.

Auch in den neuen Ländern scheint die obrigkeitsstaatliche Tradition Deutschlands über den Zusammenbruch des Nationalsozialismus hinaus wirksam geblieben und durch die politischen Strukturen des SED-Regimes gefördert worden zu sein. Zwar liegen für die Jahre 1949 bis 1989 keine mit der alten Bundesrepublik vergleichbaren Daten zum Kompetenzbewußtsein der DDR-Bevölkerung vor, doch findet man in Analysen der politischen Kultur der ehemaligen DDR zahlreiche Hinweise auf die stark obrigkeitsstaatlich geprägten Einstellungen der Bevölkerung zur Politik. In einer ersten - allerdings auf Berlin begrenzten - empirischen Analyse der politischen Orientierungen der neuen Bundesbürger konnten Fuchs, Klingemann \& Schöbel (1991, S. 41) die Annahme, die lange fortdauernde Tradition einer Untertanenkultur im östlichen Teil Deutschlands beeinflusse auch heute noch die Beziehungen der Bevölkerung zum politischen System, allerdings nicht bestätigen. Überraschenderweise lag das Gefühl persönlicher Kompetenz in Ost- und Westberlin ungefähr auf dem gleichen Niveau (vgl. auch Feist, 1991, S. 29ff.). Möglicherweise standen die 1990 ermittelten Einstellungen noch stark unter dem Eindruck der demokratischen Revolution in der ehemaligen DDR und könnten sich mit einer wachsenden zeitlichen Distanz zu diesem einschneidenden Ereignis abschwächen. In der empirischen Forschung über die Konvergenz der politischen Orientierungen in den alten und neuen Ländern wird der partizipativen Dimension schon im Hinblick auf die Diskussion über die obrigkeitsstaatliche Tradition Deutschlands besondere Beachtung zu schenken sein.

Unabhängig von seiner großen Relevanz in der empirischen Wahlforschung kommt dem Konzept der Parteiidentifikation in der empirischen Analyse politischer Kultur ein eigenständiger Stellenwert zu (vgl. hierzu Abramson, 1983, S. 11ff. und S. 225ff.; Falter, 1977). Für seine Berücksichtigung in einer Analyse des Entstehens einer gesamtdeutschen Kultur sprechen neben theoretischen Überlegungen auch die zur Entwicklung der Parteiidentifikation in der Bundesrepublik vorliegenden Befunde. Ana$\log$ zum politischen Kompetenzbewußtsein scheint auch in der Beziehung der Bundesbürger zu den politischen Parteien in den sechziger und siebziger Jahren ein Umbruch eingetreten zu sein. Die Vorbehalte gegen die politischen Parteien verschwanden zwar keineswegs, jedoch kann zwischenzeitlich keine Rede mehr davon sein, daß die Deutschen parteikritischer wären als die Bevölkerung anderer westeuropäischer Staaten (vgl. Gabriel, 1992, S.120ff.). Seit einigen Jahren deuten die vorliegenden Umfragedaten auf einen Rückgang der Parteiidentifikation hin (vgl. Klingemann \& Wattenberg, 1990; Rattinger, 1993), dessen systemische Bedeutung ohne genaue Kenntnis der Rolle der Parteiidentifikation im Gesamtzusammenhang der politischen Kultur nicht interpretierbar ist.

Von Anfang an fand das Konzept der Parteiidentifikation in Untersuchungen der politischen Kultur im wiedervereinigten Deutschland Beachtung, wenn auch zumeist in seiner Bedeutung fuir den Wahlentscheid. Eine in unserem spezifischen Kontext 
interessante, wenn auch nicht zuverlässig beantwortbare Frage wurde, in einer der ersten Publikationen zum Thema „Parteiidentifikation" in den neuen Ländern von Bluck und Kreikenbom (1993) thematisiert. Die Autoren stellten in einer Lokalstudie in Jena die These auf, es habe bereits in der Vorwendezeit in der ehemaligen DDR eine "Quasiidentifikation" mit den Westparteien gegeben. Wenn in den bisherigen empirischen Untersuchungen (vgl. dazu vor allem Dittrich, 1992; Rattinger, 1993) eine valide Messung der Parteiidentifikation in den neuen Ländern gelungen ist, dann gibt es gewisse Anhaltspunkte für die generelle Gültigkeit der Annahme von Bluck und Kreikenbom. In den Einstellungen der West- und der Ostdeutschen zu den politischen Parteien zeigt sich nämlich ein erstaunliches $\mathrm{Ma} B$ an Homogenität. Es ist also durchaus vorstellbar, daß die Einstellungen zu den politischen Parteien sich bereits jetzt zu einem gemeinsamen Element der politischen Kultur Gesamtdeutschlands entwickelt haben und - in Anbetracht der Zentralität der Parteien im System der Interessenvermittlung - den Prozeß der kulturellen Integration mittel- und langfristig fördern werden (vgl. die detaillierteAnalyse der Parteiidentifikation im Beitrag von Rattinger).

\section{Perspektiven für die empirische Forschung}

Selbst bei einer Beschränkung auf die zentralen Konstrukte steht die empirische Politikwissenschaft bei der Untersuchung des Prozesses der Etablierung einer gesamtdeutschen politischen Kultur vor einer Fülle interessanter, theoretisch und praktisch gleichermaßen wichtiger Fragen. Die bisher vorliegenden, auf einzelne Aspekte der politischen Kultur in Ost- und Westdeutschland ausgerichteten Untersuchungen vermitteln kein eindeutiges - und erst recht kein vollständiges - Bild von der Ausgangslage unmittelbar nach dem Zusammenbruch der ehemaligen DDR. Die plausible Annahme, eine vierzig Jahre dauernde Sozialisation im real existierenden Sozialismus habe im östlichen Teil Deutschlands über den Regimewechsel hinaus ihre Spuren hinterlassen, wird durch die vorliegenden Erkenntnisse der empirischen Forschung nur zum Teil gestützt. Ob dieses überraschende Ergebnis auf Unzulänglichkeiten in den Erhebungsmethoden beruht oder auf eine partielle Übereinstimmung der politischen Kultur beider Teilen Deutschlands hindeutet, ist kaum zu entscheiden. In Anbetracht des raschen und unblutigen Systemwandels in Ostdeutschland ist die Annahme jedenfalls nicht abwegig, daß die Werte, Normen, Institutionen und Verhaltenserwartungen des politischen Regimes der ehemaligen DDR im Orientierungssystem der Bevölkerung nicht besonders fest verankert waren.

Als Voraussetzung für jeden weiterführenden Schritt hat die empirische Forschung den derzeitigen Zustand der politischen Kultur Gesamtdeutschlands auf der Basis eingeführter Konzepte und bewährter Meßinstrumente möglichst genau zu beschreiben. Für die neuen Bundesländer ergibt sich hieraus die Notwendigkeit, an die im Umfeld der Wende durchgeführten Untersuchungen anzuknüpfen; für die alten Bundesländer ist auf Untersuchungen aus der gesamten Nachkriegszeit Bezug zu nehmen. Nur eine kontinuierliche empirische Erforschung der zentralen Orientierungsmuster vermittelt ein detailliertes Bild vom Wandel der politischen Kultur in beiden Teilen Deutschlands. Im Hinblick auf die Theorie- und Methodendiskussion sowie die Kenntnis der 
neueren politischen Entwicklung Deutschlands gehören die Unterstützung der Demokratie sowie der sie tragenden Werte und Normen, das Vertrauen zu den politischen Institutionen, die Parteiidentifikation und das Gefühl politischer Kompetenz bzw. Effektivität zum Mindestbestand eines derartigen Forschungsprogramms.

In der Forschungspraxis stellt sich die wissenschaftliche Analyse politischer Kultur zumeist als Vergleich der Randverteilung einzelner politischer Einstellungen in einzelnen Ländern dar. Auch in unserem Zusammenhang ist ein derartiges Vorgehen wichtig; denn von der Etablierung einer gesamtdeutschen politischen Kultur kann nur dann die Rede sein, wenn die politischen Einstellungen der alten und der neuen Bundesbürger einander im Laufe der Zeit ähnlicher werden.

Obgleich die vergleichende Beschreibung der Verteilung politischer Einstellungen in Ost- und Westdeutschland wichtig ist, sollte sich die empirische Analyse nicht auf diesen Aspekt beschränken. Die Charakterisierung der politischen Kultur als Muster von Orientierungen impliziert ein gewisses Ausmaß an Kohärenz der Einzelteile. Trotz seiner großen theoretischen und praktischen Relevanz wurde das Problem der Beziehung zwischen den einzelnen Elementen des Orientierungssystems von der empirischen Forschung weitgehend vernachlässigt. Seine Bedeutsamkeit läßt sich an Hand einiger Beispiele illustrieren. DemWandel der Parteiidentifikation oder des Regienungsvertrauens kommt eine unterschiedliche politische Tragweite $\mathrm{zu}$, je nachdem ob diese Veränderungen mit einem Wandel der Systemunterstützung verbunden sind oder nicht. Auch bei der Abschätzung der Verhaltensrelevanz politischer Unzufriedenheit ist die Klärung der Frage nicht unwichtig, ob diese Einstellung vor allem bei den politisch interessierten, informierten und handlungsbereiten Bürgerinnen und Bürgern auftritt. Nicht allein die Wechselbeziehung zwischen den einzelnen Elementen des individuellen Orientierungssystems ist für die empirische Analyse von Interesse, sondern auch die Prüfung ihrer Stabilität.

Den meisten Analysen der Bedingungen und Folgen des Auftretens bestimmter Orientierungsmuster liegt implizit ein modernisienungstheoretischer Ansatz zu Grunde, in dem der sozio-ökonomische Entwicklungsgrad eines Landes als Determinante seiner politischen Kultur angenommen wird, die ihrerseits wieder die Performanz und Stabilität des politischen Regimes beeinflussen soll (vgl. z.B. Lijphart, 1980). Im Bezugsrahmen dieses Erklärungsansatzes war es möglich, die Etablierung einer demokratischen politischen Kultur in der Nachkriegsbundesrepublik zu interpretieren und die Stabilität der bundesdeutschen Demokratie zu erklären. Der Aufbau diffuser Systemunterstützung wurde aber nicht allein auf das Wirtschaftswunder und die sozioökonomische Modernisierung der Gesellschaft, sondern auch auf den Generationenwandel und das Entstehen eines Elitenkonsenses zurückgeführt (vgl. u.a. Baker, Dalton \& Hildebrandt, 1981; Conradt, 1980). Als wichtigste Ursachen der Diffusion partizipativer Orientierungen wird man die Verbesserung der Bildungschancen und die Ausbreitung postmaterialistischer Wertorientierungen ansehen können (vgl. ausführlich Gabriel, 1986, 1987).

Über die Frage, ob die Demokratie im wiedervereinigten Deutschland auf einer stabilen kulturellen Grundlage steht, läßt sich wegen des Fehlens einer empirisch bewährten Theorie demokratischer Stabilität allenfalls spekulieren; denn die empirische Forschung ist derzeit nicht in der Lage, den makropolitischen Teil des Forschungs- 
programmes „politische Kultur“ einzulösen. Dies gilt auch für Aussagen über die kulturellen Grundlagen demokratischer Stabilität und Performanz im wiedervereinigten Deutschland. Beim derzeitigen Stand der Theoriebildung bleiben Aussagen über die Stabilität und Leistungsfähigkeit demokratischer Regime spekulativ und stark von den normativen Prämissen des Beobachters beeinflußt.

Gegenüber den makroanalytischen Möglichkeiten des Forschungsprogramms ,politische Kultur" ist Skepsis am Platze. Bei einer Konzentration der Forschungsaktivitäten auf die Mikro-Ebene, die Beschreibung und Erklärung des Verhältnisses der Bevölkerung Ost- und Westdeutschlands zum politischen System und seinen Teilsystemen steht die empirische Politikforschung allerdings vor zahlreichen spannenden Forschungsaufgaben.

\section{Literatur}

Abramowitz, Alan 1. (1980). The United States: Political Culture under Stress. In: Almond, G. \& Verba, S. (Hrsg.). The Civic Culture Revisited. An Analytic Study. Boston, S. 177-211.

Abramson, Paul A. (1983). Political Attitudes in America. Formation and Change. San Francisco. Almond, Gabriel A. \& Verba, Sidney (Hrsg.) (1980). The Civic Culture Revisited.AnAnalytic Study. Boston.

Almond, Gabriel A. (1987). Politische Kulturforschung - Rückblick und Ausblick. In: Berg-Schlosser, D. \& Schissler, J. (Hrsg.). Politische Kultur in Deutschland. Bilanz und Perspektiven der Forschung. Sonderheft 18 der PVS. Opladen, S. 27-38.

Baker, Kendall L.; Dalton, Russell J. \& Hildebrandt, Kai (1981). Germany Transformed: Political Culture and the New Politics. Cambridge, Mass.

Bauer, Petra (1991a). Politische Orientierungen im Übergang. Eine Analyse politischer Einstellungen der Bürger in West- und Ostdeutschland 1990/1991. In: Kölner Zeitschrift für Soziologie und Sozialpsychologie, S. 433-453.

Beer, Samuel H. (1973). Modern Political Development. In: Beer, S.H. \& Ulam,A.B. (Hrsg.).Patterns of Government. The Major Political Systems of Europe. 3. Auflage, New York, S. 3-118.

Bluck, Carsten \& Kreikenbohm, Henry (1993). Quasiparteibindung und Issues für Mehrdimensionalität und EinfluBfaktoren im Wahlverhalten Jenaer Bürger. In: Gabriel, O.W. \& Troitzsch, K.G. (Hrsg.). Wahlen in Zeiten des Umbruchs. Frankfurt/M. u. a., S. 455-470.

Campbell, Angus et al. (1960). The American Voter. New York.

Conradt, David P. (1980). Changing German Political Culture. In: Almond, G.A. \& Verba, S. (Hrsg.). The Civic Culture Revisited. An Analytic Study. Boston, S. 212-272.

Conradt, David P. (1991). From Outputorientation to Regime Support: Changing German political Culture. In: Hoffmann-Lange, U. (Hrsg.). Social and Political Structures in West Germany. From Authoritarianism to post-industrial Democracy. Boulder, Col., S. 127-142.

Dahl, Robert A. (1971). Polyarchy. Participation and Opposition. New Haven, London.

Dias, Patrick V. (1971). Der Begriff „Politische Kultur" in der Politikwissenschaft. In: Oberndörfer, D. (Hrsg.). Systemtheorie, Systemanalyse, Entwicklungsländerforschung. Berlin, S. 409-448.

Dittrich, Karl-Heinz (1992). Das Bild der Parteien im vereinten Deutschland. Für welche Bevölkerungsgruppen setzen sie sich ein? In: Aus Politik und Zeitgeschichte, B 34-35, S. 26-35.

Döhring, A. (1990). Aspekte des Vertrauens in Institutionen. In: Zeitschrift für Soziologie 19(2), S. 73-89

Easton, David (1975). A Reassessment of the Concept of Political Support. In: British Journal of Political Science, 5, S. 435-457.

Easton, David (1979). A Systems Analysis of Political Life. 2. Aufl., Chicago, London. 
Falter, Jürgen W. (1972). Ein Modell zurAnalyse individuellen politischen Verhaltens. In: Politische Vierteljahresschrift, 13, S. 547-566.

Falter, Jürgen W. (1977). Einmal mehr: Läßt sich das Konzept der Parteiidentifikation auf deutsche Verhältnisse übertragen? Theoretische, methodologische und empirische Probleme einer Validierung des Konstrukts „Parteiidentifikation“ für die Bundesrepublik Deutschland. In: Kaase, M. (Hrsg.). Wahlsoziologie heute. Analysen aus AnlaB der Bundestagswahl 1976. Politische Vierteljahresschrift, 8 (2/3), Opladen, S. 476-500.

Falter, JürgenW. (1980). Die Behaviourismus-Kontroverse in der amerikanischen Politikwissenschaft. Ein Beispiel für die Übertragung von Thomas Kuhns Theorie wissenschaftlicher Entwicklungsvorgänge. In: Topitsch, E. (Hrsg.). Logik der Sozialwissenschaften. Königsstein/Ts., S. 423-448.

Feist, Ursula (1991). Zur polituschen Akkulturation der vereinten Deutschen. In: Aus Politik und Zeitgeschichte, B 11-12, S. 21-32.

Fuchs, Dieter (1989). Die Unterstützung des politischen Systems der Bundesrepublik Deutschland. Opladen.

、 Fuchs, Dieter, Klingemann, H.-D. \& Schölzel, Caroline (1991). Perspektiven der politischen Kultur im vereinten Deutschland. In: Aus Politik und Zeitgeschichte, B. 22, S. 32-45.

Gabriel, OscarW. (1986). Politische Kultur, Postmaterialismus und Materialismus in der Bundesrepublik Deutschland. Opladen.

Gabriel, Oscar W. (1987a). Wahrnehmung der Politik durch den Bürger als Herausforderung für die Politikvermittlung. Vertrauen und EffektivitätsbewuBtsein als Gegenstand der politischen Kulturforschung. In: Sarcinelli, U. (Hrsg.).Politikvermittlung. Beiträge zur politischen Kommunikationskultur. Stuttgart, S. 46-70.

Gabriel, Oscar W. (1987b). Demokratiezufriedenheit und demokratische Einstellungen in der Bundesrepublik Deutschland. In: Aus Politik und Zeitgeschichte, B 22, S. 32-45.

Gabriel, Oscar W. (1990). Demokratische Entwicklung und politische Kompetenz. Eine vergleichende Analyse des KompetenzbewuBtseins der bundesdeutschen und amerikanischen Bevölkerung. In: Aus Politik und Zeitgeschichte, B 25, S. 15-26.

Gabriel, Oscar W. (1992), unter Mitarbeit von Brettschneider, Frank (Hrsg.). Die EG-Staaten im Vergleich. Strukturen, Prozesse, Politikinhalte. Opladen.

Gabriel, Oscar W. (1993). Institutionenvertrauen in Ost- und Westdeutschland. In: Aus Politik und Zeitgeschichte.

Gluchowski, Peter \& Zelle, Carsten (1992). Demokratisierung in Ostdeutschland. Aspekte der politischen Kultur in der Periode des Systemwechsels. In: Gerlich, P. ; Plasser, F. \& Ulram, P. (Hrsg.). Regimewechsel. Demokratische und politischen Kultur in Ost- und Mitteleuropa. Wien, Köln, Graz, S. 232-274.

Grunenberg, Antonia (1989). BewuBtseinslagen und Leitbilder in der DDR. In: Weidenfeld, W. \& Zimmermann, H. (Hrsg.). Deutschland Handbuch. Eine doppelte Bilanz 1949-89. München, S. 221-238.

Iwand,Wolf Dieter (1985).Paradigma Politische Kultur. Konzepte, Methoden. Engebnisse der Political Culture Forschung in der Bundesrepublik. Ein Forschungsbericht. Opladen.

Jesse, Eckard (1992). Der innenpolitische Weg zur deutschen Einheit. Zäsuren einer atemraubenden Entwicklung. In: Jesse, E. \& Mitter, A. (Hrsg.). Die Gestaltung der deutschen Einheit. Geschichte, Politik, Gesellschaft. Bonn, S. 111-141.

Kaase, Max (1983). Sinn und Unsinn des Konzepts Politische Kultur für die Vergleichende Politikwissenschaft, oder auch: Der Versuch, einen Pudding an die Wand zu nageln. In: Kaase, M \& Klingemann, H.-D. (Hrsg.). Wahlen und Wähler. Analysen aus Anlaß der Bundestagswahl 1980. Opladen, S. 144-171.

Kavanagh, Dennis (1980). Political Culture in Great Britain: The Decline of the Civic Culture. In: Almond, G.A. \& Verba, S. (Hrsg.).The Civic Culture Revisited.AnAnalytic Study. Boston, S. 124176.

Klingemann, Hans Dieter \& Wattenberg, Martin P. (1990). Zerfall und Entwicklung von Parteien- 
systemen: Ein Vergleich der Vorstellungsbilder von den politischen Parteien in den Vereinigten Staaten von Amerika und der Bundesrepublik Deutschland. In: Kaase, M. \& Klingemann, H.-D. (Hrsg.). Wahlen und Wähler:Analysen ausAnlaß der Bundestagswahl 1987. Opladen, S. 325-345. Lijphart, Arend (1980). The Structure of Inference. In: Almond, G.A. \& Verba, S. (Hrsg.). The Civic Culture Revisited. An Analytic Study. Boston, S. 37-56.

Lipset, Seymour M. (1969). Some Social Requisites of Democracy. In: Cnudde, C. \& Neubauer, D.E. (Hrsg.). Empirical Democratic Theory. Chicago, S. 151-192.

Lipset, Seymour M. \& Schneider, William (1983). The Confidence Gap. Bussiness, Labor, and Government in the Public Mind, New York. London.

Marsh, Alan (1977). Protest and Political Consciousness. Beverly Hills, London.

Miller, Warren E.\& Traugott, Santa A. (1989).American National Election Studies Data Sourcebook, 1912-1986. Cambridge, Mass., London.

Muller, Edward N. \& Jukam, Thomas O. (1977). On the Meaning of Political Support. In: American Political Science Review, 71, S. 1561-1595.

Pappi, Franz Urban (1986). Politische Kultur. Forschungsparadigma, Fragestellungen, Untersuchungsmöglichkeiten. In: Kaase, M. (Hrsg.). Politische Wissenschaft und politische Ordnung. Analysen zur Theorie und Empirie demokratischer Regierungsweise. Festschrift zum 65. Geburtstag von Rudolf Wildenmann. Opladen, S. 279-291.

Parry, Geraint (1976). Trust, Distrust and Consensus. In: BJPS, 6, S. 129-142.

Parsons, Talcott \& Shills, Edward (1962). Values,Motives, and Systems of Action. In: Parsons, T. et al. 1962b. New York, Evanston, S. 47-275.

Patrick, Glenda M. (1984). Political Culture. In: Sartori, G. (Hrsg.). Social Science Concepts: A Systematic Analysis. London, S. 265-314.

Powell, G. Bingham (1983). Contemporary Democracy. Participation, Stability, and Violence. Cambridge, Mass.

Pye, Lucian W. (1972). Culture and Political Science. Problems in the Evaluation of the Concept of Political Culture. In: Social Science Quarterly, 53, S. 285-296.

Rattinger, Hans (1993). Abkehr von den Parteien? Dimensionen der Parteiverdrossenheit. In: Aus Politik und Zeitgeschichte, B 11, S. 24-35.

Rohe, Karl (1987). Politische Kultur und der kulturelleAspekt von politischerWirklichkeit. Konzeptionelle und typologische Überlegungen zu Gegenstand und Fragestellung politischer Kulturforschung. In: Berg-Schlosser, D. \& Schissler, J. (Hrsg.). Politische Kultur in Deutschland. Bilanz und Perspektiven der Forschung. Sonderheft 18 der PVS. Opladen, S. 39-48.

Rohe, Karl \& Dörner, Andreas (1990). Von der Untertanenkultur zur „Partizipationsrevolution“? Kontinuität und Wandel politischer Kultur in Deutschland. In: Politische Bildung, 23, S. 18-33.

Snidermann, Paul M. (1981). A Question of Loyality. Berkeley u.a.

Sontheimer, Kurt (1989). Grundzüge des politischen Systems der Bundesrepublik Deutschland. München.

Thompson, Denis F. (1990). The Democratic Social Science and Democratic Theory in the Twentieth Century. Cambridge.

Topf, Richard G. (1989). Political Change and Political Culture in Britain, 1959-1987. In: Gibbins, J. (Hrsg.). Contemporary Political Culture. Politics in a PostmodernAge. London et al., S. 52-80.

Verba, Sidney (1965). Conclusion: Comparative Political Culture. In: Pye, L.W. \& Verba, S. (Hrsg.). Political Culture and Political Development. Princeton, N.J., S. 512-560.

Verba, Sidney (1965). Germany: The Remaking of a Political Culture. In: Pye, L.W. \& Verba, S. (Hrsg.). Political Culture and Political Development. Princeton, N.J., S. 130-170.

Westle, Bettina (1989).Politische Legitimität. Theorien, Konzepte, empirische Befunde. Baden-Baden.

Wright, James D. (1976). The Dissent of the Governed. Alienation and Democracy in America. New York, San Francisco, London.

ZUMA (1983). Handbuch Sozialwissenschaftlicher Skalen. Mannheim: Zentrum für Umfragen, Methoden und Analysen e. V., Bonn: Informationszentrum Sozialwissenschaften. 